EPiC Series in Computing
Volume 63, 2019, Pages 182-188
Proceedings of 32nd International Conference on
Computer Applications in Industry and Engineering

\title{
A Novel Approach Toward Designing A Non- DHT Based Structured P2P Network Architecture
}

\author{
Indranil Roy, Bidyut Gupta, Banafsheh Rekabdar, and Henry Hexmoor \\ Department of Computer Science \\ Southern Illinois University \\ Carbondale, IL, USA \\ Indranil.roy@siu.edu, \{bidyut, brekabdar,hexmoor\}@cs.siu.edu
}

\begin{abstract}
In this paper, we have applied modular arithmetic, specifically residue class (RC), to design a non-DHT-based structured P2P network. It is an interest based tree architecture. It is known as pyramid tree. A node $i$ in this tree represents a group of peers that are interested in a particular resource of type $i$. It is not a conventional tree. In the present work, such a P2P architecture has been the choice because in a complete pyramid tree, multiple paths exist between most of its nodes. Such a structural characteristic can be helpful from the viewpoints of designing load balanced as well as robust communication protocols. Besides, search latency for its intergroup data lookup algorithm is bounded by the tree diameter and is independent of the number of the distinct resource types as well as the total number of peers present in the system. In addition, any intra-group data look up communication needs only one overlay hop.
\end{abstract}

Index Terms - Structured P2P network, Residue class, pyramid tree, tree diameter

\section{INTRODUCTION}

Peer-to-Peer (P2P) overlay networks are widely used in distributed systems due to their ability to provide computational and data resource sharing capability in a scalable, self-organizing, distributed manner. P2P networks are classified into two classes: unstructured and structured ones. In unstructured systems [2] peers are organized into arbitrary topology. It takes help of flooding for data look up. Problem arising due to frequent peer joining and leaving the system, also known as churn, is handled effectively 
in unstructured systems. However, it compromises with the efficiency of data query and the much needed flexibility. In unstructured networks, lookups are not guaranteed. On the other hand, structured overlay networks provide deterministic bounds on data discovery. They provide scalable network overlays based on a distributed data structure which actually supports the deterministic behavior for data lookup. Recent trend in designing structured overlay architectures is the use of distributed hash tables (DHTs) [3] - [5]. Such overlay architectures can offer efficient, flexible, and robust service [3] - [5], [7], [8].

However, maintaining DHTs is a complex task and needs substantial amount of effort to handle the problem of churn. So, the major challenge facing such architectures is how to reduce this amount of effort while still providing an efficient data query service. In this direction, there exist several important works, which have considered designing hybrid systems [1], [6], [9] - [11]; these works attempt to include the advantages of both structured and unstructured architectures. However, these works have their own pros and cons.

\section{Our Contribution}

In this paper, we have considered designing interest-based P2P systems [6], [12], [13], [15]. The rationale behind this choice is that users sharing common interests are likely to share similar contents, and therefore searches for a particular type of content is more efficient if peers likely to store that content type are neighbors [14]. We have pointed out above the disadvantages of DHT-based systems. Therefore, in this paper, we have considered the problem of designing non-DHT based structured system that does not have such problems. We have used a mathematical model based on modular arithmetic, specifically residue class (RC), to design the architecture. To the best of our knowledge, there does not exist any work in this direction that has used this mathematical model. Ours is an interest based tree architecture. It is known as pyramid tree [16]. It is not a conventional tree. This tree structure found its applications in the past in the area of design for testability for VLSI chips. In the present work, a node $i$ in this tree represents a group of peers that possess a particular resource of type $i$. Hence, each node corresponds to a distinct resource type. In the present work, such a tree architecture has been the choice because in a complete pyramid tree, multiple paths exist between most of its nodes. Such a structural characteristic can be helpful from the viewpoints of designing communication protocols that are load balanced as well as robust. In this architecture, search latency for its inter-group data lookup algorithm is bounded by the tree diameter and is independent of the number of the distinct resource types as well as the total number of peers present in the system. The proposed architecture has appeared in Section II. Salient features of the architecture and an analytical comparison of data lookup complexities with some important works have appeared in Section III.

In this context, it is worth mentioning that the interest-based architecture proposed in [15] has used a different number theoretic approach, viz., Linear Diophantine Equation to design a non-DHT based hierarchical structured P2P system, which offers considerable improvement in the efficiency of the data lookup algorithms compared to some well-established DHT-based systems. The search latency for its inter-group data lookup algorithm is bounded only by the number of the distinct resource types and is independent of the total number of peers present in the system. This is its most important advantage that makes it superior to some important DHT-based systems.

\section{PROPOSED ARCHITECTURE}

In this section, we present a structured architecture for interest-based peer-to-peer system and the required mathematical basis supporting the architecture. The following notations along with their interpretations will be used while we define the architecture.

Definition 1. We define a resource as a tuple $\left\langle R_{i}, V\right\rangle$, where $R_{i}$ denotes the type of a resource and $V$ is the value of the resource. 
Note that a resource can have many values. For example, let $\mathrm{R}_{\mathrm{i}}$ denote the resource type 'songs' and $\mathrm{V}^{\prime}$ ' denote a particular singer. Thus $\left\langle\mathrm{R}_{\mathrm{i}}, \mathrm{V}^{\prime}>\right.$ represents songs (some or all) sung by a particular singer $\mathrm{V}^{\prime}$.

Definition 2. Let $S$ be the set of all peers in a peer-to-peer system. Then $S=\left\{P^{R i}\right\}, 0 \leq i \leq n-1$, where $P^{R i}$ denotes the subset consisting of all peers with the same resource type $R_{i}$. and the number of distinct resource types present in the system is $n$. Also for each subset $P^{R i}$, we assume that $P_{i}$ is the first peer among the peers in $P^{R i}$ to join the system. We call $P_{i}$ as the group-head of group $G_{i}$ formed by the peers in the subset $P^{R i}$.

We now describe our proposed architecture suitable for interest-based peer-to-peer system. We assume that no peer can have more than one resource type. Generalization of the architecture is not considered in this paper.

\section{A. Pyramid Tree}

We propose the following overlay architecture.

1) In the tree, there are n numbers of completely connected networks (groups) of peers. Each such group, say $\mathrm{G}_{\mathrm{i}}$ is formed by the peers of the subset $\mathrm{P}^{\mathrm{Ri}},(0 \leq \mathrm{i} \leq \mathrm{n}-1)$, such that all peers $\left(\epsilon \mathrm{P}^{\mathrm{Ri}}\right)$ are directly connected (logically) to each other, resulting in the network diameter of 1 . The groupheads of the $n$ groups are connected to form the edges (links) of the pyramid tree.

2) Any communication between a peer $p_{i} \in G_{i}$ and a peer $p_{j} \in G_{j}$ takes place only via the respective group-heads $\mathrm{P}_{\mathrm{i}}$ and $\mathrm{P}_{\mathrm{j}}$ and with the help of tree traversal.

\section{B. Relevant Properties of Modular Arithmetic}

Consider the set $S_{n}$ of nonnegative integers less than $n$, given as $S_{n}=\{0,1,2, \ldots$ (n-1)\}. This is referred to

as the set of residues, or residue classes $(\bmod n)$. That is, each integer in $S_{n}$ represents a residue class (RC). These $(\bmod n)$.

residue classes can be labelled as [0], [1], [2], .., [n-1], where [r] $=\{a$ : a is an integer, a $\equiv r$

For example, for $\mathrm{n}=3$, the classes are:

$$
\begin{aligned}
& {[0]=\{\ldots .,-6,-3,0,3,6, \ldots\}} \\
& {[1]=\{\ldots .-5,-2,1,4,7, \ldots\}} \\
& {[2]=\{\ldots .-4,-1,2,5,8, \ldots\}}
\end{aligned}
$$

Thus, any class $r(\bmod n)$ of $S_{n}$ can be written as follows:

$$
[r]=\{\ldots,(\mathrm{r}-2 \mathrm{n}),(\mathrm{r}-\mathrm{n}), \mathrm{r},(\mathrm{r}+\mathrm{n}),(\mathrm{r}+2 \mathrm{n}), \ldots,(\mathrm{r}+(\mathrm{j}-1) \cdot \mathrm{n}),(\mathrm{r}+\mathrm{j} \cdot \mathrm{n}),(\mathrm{r}+(\mathrm{j}+1) \cdot \mathrm{n}), \ldots . .\}
$$
logical

In the proposed $\mathrm{P} 2 \mathrm{P}$ architecture, we shall use the numbers belonging to different classes as the

addresses of the peers; therefore, for the sake of simplicity we shall use only the positive integer values. Before we 
propose the mechanism of logical address assignments, we state the following relevant property of residue class.

Lemma 1. Any two numbers of any class $r$ of $S_{n}$ are mutually congruent.

Proof. Let us consider any two numbers $\mathrm{N}^{\prime}$ and $\mathrm{N}^{\prime \prime}$ of class $\mathrm{r}$. these numbers can be written as

$$
\mathrm{N}^{\prime} \equiv \mathrm{r}(\bmod \mathrm{n}) \text {; therefore, }\left(\mathrm{N}^{\prime}-\mathrm{r}\right) / \mathrm{n}=\text { an integer, say I' }
$$

and $\mathrm{N}^{\prime \prime} \equiv \mathrm{r}(\bmod \mathrm{n})$; therefore, $\left(\mathrm{N}^{\prime \prime}-\mathrm{r}\right) / \mathrm{n}=$ an integer, say I"

Using (1) and (2) we get the following, $\left(\mathrm{N}^{\prime}-\mathrm{N}^{\prime \prime}\right) / \mathrm{n}=\left(\left(\mathrm{N}^{\prime}-\mathrm{r}\right)-\left(\mathrm{N}^{\prime \prime}-\mathrm{r}\right)\right) / \mathrm{n}=\mathrm{I}^{\prime}-\mathrm{I}^{\prime \prime}=$ an integer.

Therefore, $\mathrm{N}^{\prime}$ is congruent to $\mathrm{N}^{\prime \prime}$; that is, $\mathrm{N}^{\prime} \equiv \mathrm{N}^{\prime \prime}(\bmod \mathrm{n})$; also, $\mathrm{N}^{\prime \prime} \equiv \mathrm{N}^{\prime}(\bmod \mathrm{n})$ because congruence relation $(\equiv)$ is symmetric. Hence, the proof. $\square$

\section{Assignments of Overlay Addresses}

Assume that in an interest-based $\mathrm{P} 2 \mathrm{P}$ system there are $\mathrm{n}$ distinct resource types. Note that $\mathrm{n}$ can be set to an extremely large value a priori to accommodate large number of distinct resource types. Consider the set of all peers in the system given as $\mathrm{S}=\left\{\mathrm{P}^{\mathrm{Ri}}\right\}, 0 \leq \mathrm{i} \leq \mathrm{n}-1$. Also as mentioned earlier, for each subset $P^{R i}$ (i.e. group $G_{i}$ ) peer $P_{i}$ is the first peer with resource type $R_{i}$ to join the system.

In the proposed overlay architecture, the positive numbers belonging to different classes are used to define the following:

(a) Logical addresses of peers in a subnet $\mathrm{P}^{\mathrm{Ri}}$ ( i.e. group $\mathrm{G}_{\mathrm{i}}$ ). Use of these addresses will be shown to justify that all peers in $G_{i}$ are directly connected to each other (logically) forming an overlay network of diameter 1. In graph theoretic term, each $\mathrm{G}_{\mathrm{i}}$ is a complete graph.

(b) Identifying the edges (links) that connect different group-heads of the $\mathrm{n}$ groups in the tree.

The assignment of logical addresses to the peers in the groups and the resources happen as follows:

1) Each group-head $P_{r}$ of group $G_{r}$ is assigned with the minimum nonnegative number $(r)$ of residue class $r(\bmod n)$ of the residue system $\mathrm{S}_{\mathrm{n}}$.

2) All peers having the same resource type $R_{r}$ will form the group $G_{r}$ (i.e. the subset $P^{R r}$ ) with group-head $P_{r}$. Each new peer joining group $G_{r}$ is given the group membership address $(r+$ j.n), for $\mathrm{j}=0,1,2, \ldots$

3) Resource type $\mathrm{R}_{\mathrm{r}}$ possessed by peers in $\mathrm{G}_{\mathrm{r}}$ is assigned the code $r$ which is also the logical address of the group-head $\mathrm{P}_{\mathrm{r}}$ of group $\mathrm{G}_{\mathrm{r}}$.

An example of a complete pyramid tree is shown in Fig. 1. In the figure, assignments of the overlay addresses of the group- heads are done in the following way. A group-head $\mathrm{P}_{0}$ with resource type ' 0 ' is assigned the address ' 0 ' if it is the first peer to join the network, thereby becoming the root node of the tree. In general, suppose that there exist already i number of group-heads (i.e. $\left.\mathrm{P}_{0}, \mathrm{P}_{1}, \ldots \mathrm{P}_{\mathrm{i}-1}\right)$ in the tree. Then the next peer joining the system as the group-head with resource type $\mathrm{i}$ will be assigned with the address i. For example, the sixth group-head joining the system will have the logical address as 5 and the code of the resource type it possesses is 5 . The edges are formed according to the pyramid tree structure. Observe that no node can have a degree larger than 4 and only two nodes 6 and 9 at the leaf level have degree one each. Thus, the architecture offers multiple paths between most of its nodes. 


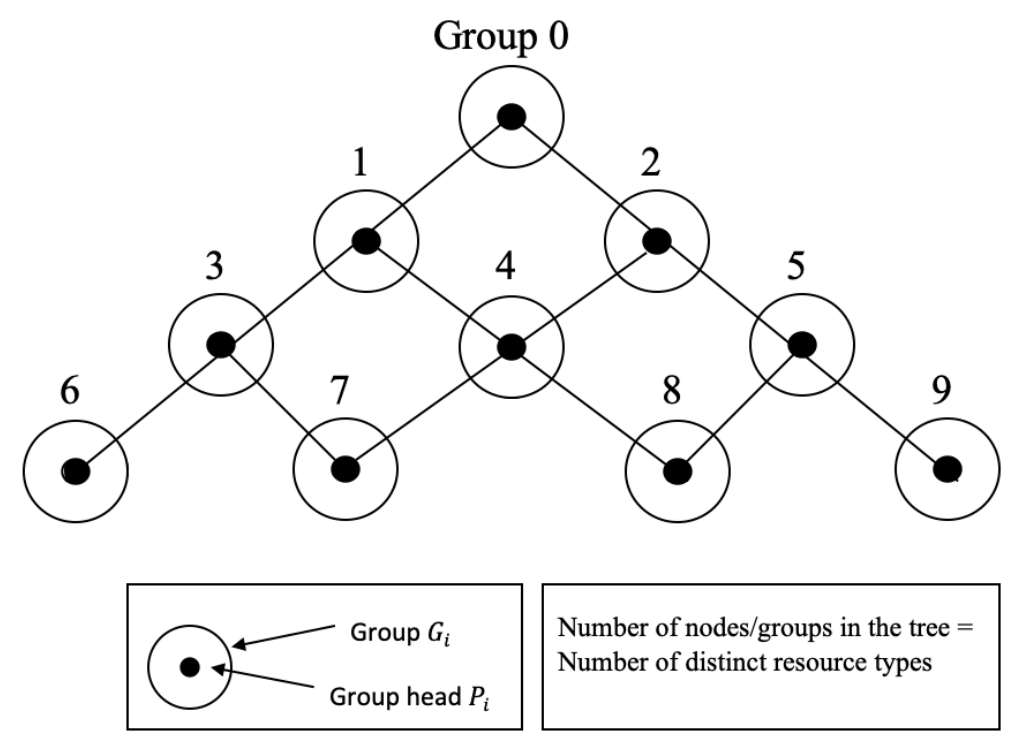

Fig. 1. A 3-level complete pyramid tree.

Definition 3. Two peers of a group $G_{r}$ are logically linked together if their assigned logical addresses are mutually congruent.

Lemma 2. Each group $G_{r}$ forms a complete graph.

Proof. According to Definition 3, two peers of a group $\mathrm{G}_{\mathrm{r}}$ are logically linked together if their assigned logical addresses are mutually congruent. Also from Lemma 1, we note that any two numbers of any class $r$ of $S_{n}$ are mutually congruent. Therefore, every peer has direct logical connection with every other peer in the same group $\mathrm{G}_{\mathrm{r}}$. Hence, the proof.

Observation 1. Any intra-group data look up communication needs only one overlay hop.

Observation 2. Search latency for inter-group data lookup algorithm is bounded by the diameter of the tree.

\section{SALIENT FEATURES OF THE ATCHITECTURE}

We summarize below the salient features of the proposed architecture.

1. Use of modular arithmetic allows a group-head address to be identical to the code used to identify the resource type owned by the group.

2. Each overlay group is completely connected. That is, in graph theoretic term it is a complete graph consisting of the peers in the group. Therefore, its diameter is just 1. Because of this smallest possible diameter (in terms of number of overlay hops), the architecture offers minimum search latency inside a group. 
3. In a complete pyramid tree, multiple paths exist between most of its nodes. Such a structural characteristic can be helpful from the viewpoints of designing communication protocols that are load balanced as well as robust.

4. Search latency for inter-group data lookup algorithm is bounded by the tree diameter and is independent of the number of the distinct resource types as well as the total number of peers present in the system.

\section{Data Lookup Complexity}

In Table 1, we have presented an analytical comparison of data lookup complexities with some important works.

Table 1 Data Lookup Complexity Comparison

\begin{tabular}{|c|c|c|c|c|}
\hline & CAN & Chord & Pastry & Our Work \\
\hline Architecture & DHT-based & DHT-based & DHT-based & Residue Class (RC)-based \\
\hline Lookup Protocol & $\begin{array}{l}\{\text { Key, value }\} \text { pairs to } \\
\text { map a point } \mathrm{P} \text { in the } \\
\text { coordinate space using } \\
\text { uniform hash function. }\end{array}$ & $\begin{array}{l}\text { Matching key and } \\
\text { NodeID. }\end{array}$ & $\begin{array}{l}\text { Matching key and } \\
\text { prefix in NodeID. }\end{array}$ & $\begin{array}{c}\text { Inter-Group: } \\
\text { Routing through } \\
\text { Group-heads, } \\
\text { Intra-group: } \\
\text { Complete Graph }\end{array}$ \\
\hline Parameters & $\begin{array}{c}N \text {-number of peers in } \\
\text { network, } \\
d \text {-number of } \\
\text { dimensions. }\end{array}$ & $\begin{array}{c}N \text {-number of } \\
\text { peers in network. }\end{array}$ & $\begin{array}{l}\text { N-number of peers } \\
\text { in network, } \\
b \text {-number of bits ( } B \\
=2^{b} \text { ) used for the } \\
\text { base of the chosen } \\
\text { identifier. }\end{array}$ & $\begin{array}{c}n \text { - Number of distinct } \\
\text { resource types, } \\
\mathrm{N} \text { - number of peers in } \\
\text { network, } \\
n<<N \\
\text { t - tree diameter }<n<<N\end{array}$ \\
\hline $\begin{array}{c}\text { Lookup } \\
\text { Performance }\end{array}$ & $O\left(\mathrm{~d} \mathrm{~N}^{1 / \mathrm{d}}\right)$ & $O(\log \mathrm{N})$ & $O\left(\log _{\mathrm{B}} \mathrm{N}\right)$ & $\begin{array}{c}\text { Inter-Group: } \\
\qquad(\mathrm{t}) \\
\text { Intra-group: } \\
O(1)\end{array}$ \\
\hline
\end{tabular}

\section{CONCLUSION}

In this paper, we have presented a new non-DHT based structured P2P architecture. We have applied some property of modular arithmetic, specifically residue class (RC), to design a scalable, structured overlay P2P system, which provides highly efficient data lookup algorithms. One noteworthy point is that search latency for inter-group data lookup is a function of the diameter of the tree and is independent of the total number of peers in the system unlike in DHT-based systems. In addition, any intra-group data look up communication needs only one overlay hop. Beside, existence of multiple paths between most nodes can help in designing communication protocols that are load balanced as well as robust. 
The present work is part of an ongoing research project; future work is directed at consideration of incomplete pyramid tree and possible further reduction in inter-group search latency.

\section{REFERENCES}

[1] P. Ganesan, Q.Sun, and H. Garcia-Molina, "Yappers: A peer-to-peer lookup service over arbitrary topology," Proc. IEEE Infocom 2003, San Francisco, USA, March 30 - April 12003.

[2] Y. Chawathe, S. Ratnasamy, L. Breslau, N. Lanham, and S. Shenker, "Making gnutella-like p2p systems scalable," Proc. ACM SIGCOMM, Karlsruhe, Germany, August 25-29 2003.

[3] B. Y. Zhao,L. Huang, S. C. Rhea, J. Stribling, A. Zoseph, and J. D. Kubiatowicz, "Tapestry: A Global-Scale Overlay for Rapid Service Deployment", IEEE J-SAC, vol. 22, no. 1, pp. 41-53, Jan. 2004.

[4] A. Rowstron and P. Druschel, "Pastry: Scalable, Distributed Object Location and Routing for Large Scale Peer-to-Peer Systems", Proc. FIP/ACM Intl. Conf. Distributed Systems Platforms (Middleware), pp. 329-350, 2001.

[5] I. Stocia, R. Morris, D. Liben-Nowell, D. R. Karger, M. Kaashoek, F. Dabek, and H. Balakrishnan, "Chord: A Scalable Peer-to-Peer Lookup Protocol For Internet Applications”, IEEE/ACM Tran. Networking, vol. 11, No. 1, pp. 17-32, Feb. 2003.

[6] M. Yang and Y. Yang, "An Efficient Hybrid Peer-to-Peer System for Distributed Data Sharing”, IEEE Trans. Computers, vol. 59, no. 9, pp. 1158-1171, Sep. 2010.

[7] M. Xu, S. Zhou, and J. Guan, “A New and Effective Hierarchical Overlay Structure for Peer-to-Peer Networks”, Computer Communications, vol. 34, pp. 862-874, 2011.

[8] D. Korzun and A. Gurtov,"Hierarchical Architectures in Structured Peer-to-Peer Overlay Networks", Peer-to-Peer Networking and Applications, Springer, pp. 1-37, March 2013

[9] Z. Peng, Z. Duan, J.Jun Qi, Y. Cao, and E. Lv, “HP2P: a hybrid hierarchical p2p network,” Proc. Intl. Conf. Digital Society, 2007.

[10] K. Shuang, P Zhang, and S. Su, "Comb: a resilient and efficient two-hop lookup service for distributed communication system,” Security and Communication Networks, vol. 8(10), pp. 1890-1903, 2015.

[11] M. Kleis, E. K. Lua,, and X. Zhou, “ Hierarchical Peer-to-Peer Networks using Lightweight SuperPeer Topologies,” Proc. IEEE Symp. Computers and Communications, 2005.

[12] R. Zhang and Y.C. Hu, “Assisted peer-to-peer search with partial indexing,” IEEE Trans. Parallel and Distributed Systems, vol. 18(8), pp. 1146-1158, 2007.

[13] E. Cohen, A. Fiat, H. Kaplan, “Associative search in peer-to-peer networks: harnessing latent semantics,” vol. 2, pp. 1261 $1271,2003$.

[14] Andrea Passarella, "A survey on content-centric technologies for the current internet: cdn and p2p Solutions," Computer Communications, vol. 35, pp. 1-32, 2012.

[15] Bidyut Gupta, Nick Rahimi, Shahram Rahimi, and Ashraf Alyanbaawi, "Efficient Data Lookup in Non-DHT Based Low Diameter Structured P2P Network,” Proc. IEEE $15^{\text {th }}$ Int. Conf. Industrial Informatics (IEEE INDIN), July 2017, Emden, Germany.

[16] Bidyut Gupta and Mohammad Mohsin, "Fault-Tolerance in Pyramid Tree Network Architecture," J. Computer Systems Science and Engineering, Vol. 10, No. 3, pp. 164-172, July, 1995. 